\title{
COMETS AS A SOURCE OF INTERPLANETARY AND INTERSTELLAR GRAINS
}

\author{
F. Hoyle and N.C. Vickramasinghe \\ School of Vathematics, \\ University of Wales College of Cardiff, \\ Senghenydd Road, \\ Cordiff, CF2 $4 A G$ \\ Vales, U.I.
}

ABSTRACT. Properties of cometary dust with regard to bulk density, optical characteristics and sizes, derived from recent observations, are used to model scattering properties of cometary and interstellar grains. A wide range of astronomical observations are shown to be explained if cometary objects are hypothesised as a major source of dust grains in the galaxy.

\section{Introduction}

Although a general similarity between cometary, interplanetary and interstellar dust had been recognised for some time, more precise comparisons were made possible only after the recent explorations of Comet Halley. Resemblances that are more than casual with regard to composition, sizes or optical characteristics would point in the direction of a common origin for the three types of dust. Ve shall argue in the present paper that strong similarities in properties do indeed exist and that they could be interpreted to imply a causal link in the direction from comets to interstellar grains.

The connection between interplanetary dust and comets is accepted without dispute. Whipple (1967) estimated that a dust injection rate from comets amounting to $\sim 10^{7} \mathrm{~g} \mathrm{~s}^{-1}$ is needed to maintain the zodiacal dust cloud. Dust particles in this cloud, with typical sizes $1-10 \mu \mathrm{m}$, spiral inwards towards the sun due to Poynting-Robertson effect and the entire cloud would be drained on a timescale of $\sim 10^{4}$ yr. $\quad 1$ replenishment of the cloud by the release of dust from short-period comets seems inadequate, the rate of injection falling below the requirements of $\sim 10^{7} \mathrm{~g} \mathrm{~s}-1$ by an order of magnitude (Rösser, 1976). A way out of the difficulty may be to suggest that a contribution arises from long-period comets by an unknown orbital mechanism (Delsemme, 1976), or from a population of dormant short-period comets with fragile dark surfaces (Hoyle and Vickramasinghe, 1986).

$\Lambda$ fraction of the dust released from long-period comets might be expected to be expelled into the interstellar medium by the action of

A.C. Levasseur-Regourd and H. Hasegawa (eds.), Origin and Evolution of Interplanetary Dust, $235-240$.

(c) 1991 Kluwer Academic Publishers, Printed in Japan. 
radiation pressure from the Sun. However, the present day injection rate from sun-like stars would not suffice to contribute significantly to the mass of interstellar dust. Although the mass of comets currently resident in the Dort cloud is estimated at $\sim 10^{29} \mathrm{~g}$, the total mass of cometary material expelled from the entire solar system at the stage when the planets Uranus and Neptune were being accumulated could have been as high as $\sim 10^{30} \mathrm{~g}$. With an estimated 1011 candidate stars possessing sun-1ike histories, the mass of cometary dust injected into the ISI is $\sim 10^{41} \mathrm{~g}$, which is close to the total mass of the interstellar grains in the Galaxy.

\section{Structure and Size Distribution of Dust}

Cometary particles associated with meteor streams such as the Geminids and Giacobinids are known to be characterised by low values of the bulk density ranging from $\sim 1-10^{-2} \mathrm{~g} \mathrm{~cm}^{-3}$ (Millman, 1976). Similar 10w values of density were also inferred from studies of photographic and radio meteors. Hore recently, direct sampling of cometary dust in the upper atmosphere has provided additional evidence of $10 \mathrm{w}$ density aggregates of particles with overall compositions similar to carbonaceous chondritic material (Brownlee, 1978). Furthermore, in situ studies of dust from Comet Halley have yielded evidence of low average values of the bulk density, $\sim 0.1 \mathrm{~g} \mathrm{~cm}^{-3}$, with a high proportion of grains involving the elements $\mathrm{C}, \mathrm{H}, \mathrm{N}, \mathrm{N}$ in the form of complex organic molecules (Kissell et al. 1986; Kissell and Krueger, 1987).

Data from the Giotto Dust Impact Detection System (DIDSY) provides important information on the mass distribution function of cometary grains (McDonnell et al., 1987). The determination of a size distribution from this data demands a knowledge of both the shape of grains as well as the bulk density. Assuming a constant value of the bulk density, and a spherical shape, the main features of the particle size distribution may be represented by the power law:

$$
\mathrm{n}(\mathrm{a}) \mathrm{da}=\text { const. } \mathrm{a}^{-3.6} \quad \mathrm{a}_{1}<\mathrm{a}<\mathrm{a}_{2}
$$

The upper and lower bounds $a_{2}, a_{1}$ are dependent on the precise value of the average bulk density, a parameter which is at present somewhat uncertain. It is possible that this density could vary systematically with the radius a. In view of the uncertainties that are involved here we shall leave $a_{1}$ as a free parameter to be fitted to a given observational situation, except that to within an order of magnitude we require $a_{1} \sim 0.1 \mu \mathrm{m}$. The appropriate upper limit $a_{2}$ in most applications is $\sim 10^{2} \mu \mathrm{m}$, corresponding to masses $\sim 1 \mu \mathrm{g}$ above which the size spectrum deviates from equation (1) (HcDonnell et al., 1987). In problems where the upper end of the size spectrum has a dominant influence we adopt a refinement to (1) suggested by the data of Mazets et al. (1986). In the discussion that follows, we shall refer to such a modification as "the cometary size distribution". 


\section{Scattering Efficiency Factor of Cometary Dust}

If a large fraction of interstellar grains have a cometary origin the extinction of starlight should be explained on the basis of a cometary distribution of grain radii and an appropriate index of refraction. Ve have shown earlier that a particular size distribution of hollow organic grains comprised of material that possesses an average refractive index $n=1.16$ could produce a very close agreement with the visual extinction law as shown in the left hand panel of Fig. 1. The size-distribution function which is appropriate to endospore forming bacteria used for this calculation is shown in the histogram of the right hand panel. In averge refractive index $\mathrm{n}=1.16$ arises if an organic sphere of refractive index 1.4 has a vacuum cavity taking up 60 percent of the volume. The heavy curve in Fig. 1 (right hand panel) depicts the cometary distribution function (1)
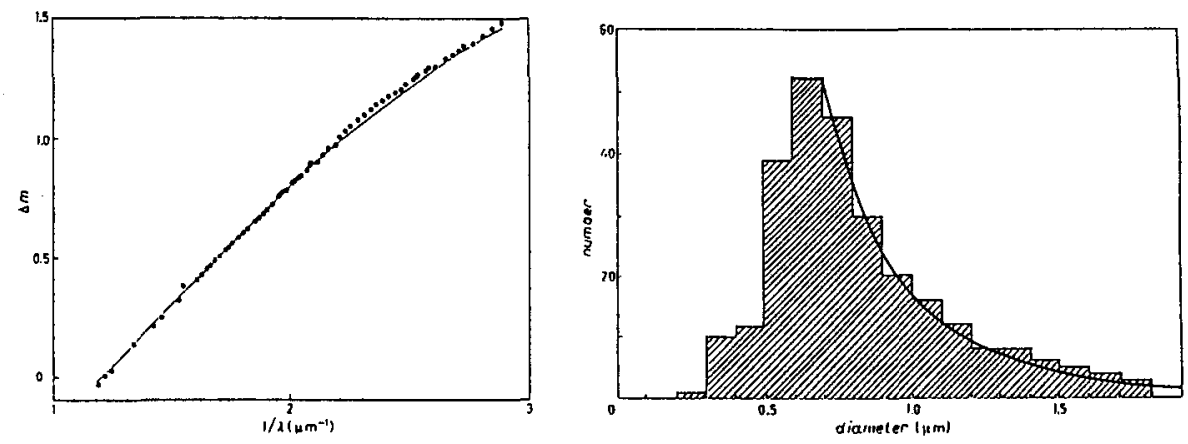

Fig. 1: (Left) Interstellar extinction data for the Cygnus region from Nandy (1964) compared with normalised extinction for a size distribution of hollow grains with average refractive index $n=1.16$. (Right) Distribution of diameters of endospore-forming bacteria (histogram) compared with the observed cometary size distribution (heavy curve).

which is seen to be in remarkable agreement with the bacterial size distribution function for $a>a_{1} \cong 0.325 \mu \mathrm{m}$. A computed extinction for this power law distribution function, assuming spheres with $m=$ 1.16 , is found to be very close to the extinction behaviour calculated in Fig. 1. The result, however, is somewhat sensitive to the assumed cut-off radius $a_{1}$, although it is insensitive to the precise value of the upper limit $a_{2}$.

\section{Optical Polarization of Comet Halley}

Whereas the average extinction cross-section for the size distribution given by (1) is mainly controlled by the smallest sizes, the integrated angular scattering properties tend to be dominated by 
larger grains. For a refractive index $m=1.4-0.05 i$ we find that the integrated intensity of scattered radiation at a typical scattering angle $\phi=60^{\circ}$ is dominated by particles of radi $\sim 15 \mu \mathrm{m}$ in the distribution function of cometary grains. Large sizes are similarly dominant in determining the polarization properties of the scattered light.

Measurements of the phase angle dependence of the polarization of scattered light from the dust coma of comet Halley when compared with the polarization calculated for a cometary size distribution of spherical-particles yields good agreement for the case of refractive index $\mathrm{m}=1.385-0.032 \mathrm{i}$ (Mukai et al., 1987; Le Borgne et al., 1986). From an exhaustive set of numerical computations, Mukai et al. (1987) have inferred that any major departures from this best value of $m$ cannot be permitted within the Iimitations of the Hie scattering model. Preliminary calculations of polarization on the basis of a hollow grain model provide a consistent explanation of the same polarization data. In this model we require a Güttler computation for hollow organic spheres with an inner to outer radius ratio $a_{0} / a=$ $(0.6)^{1 / 3}$, where a is distributed according to (1) and $\mathrm{m}$ is taken to be 1.4-0.05i. The small imaginary part of m may be interpreted as being appropriate to a trace contamination with graphitic material. The agreement with the data points is found to be of a generally similar quality to that for a filled organic sphere, as indicated in the calculation of Mukai et al.

The wavelength dependence in the range $1900<\lambda<2900 \AA$ of the total scattering function of dust in the coma of comet Halley was estimated by Vallis and Vickramasinghe (1990) from IUE spectra obtained on 14 March 1986. The observations implied a scattering angle of $135^{\circ}$. For this value of the scattering angle the normalised scattering function for a cometary size distribution of hollow organic grains has been found to fit the data.

\section{Infrared Properties of Cometary and Interstellar Dust}

The first announcement by Vickramasinghe and Allen (1986) of a broad emission feature centred at $3.4 \mu \mathrm{m}$ provided decisive evidence on the complex organic nature of the dust from comet Halley. This data was originally modelled in a simple way using a single grain temperature $\mathrm{T}$ to yield a flux

$$
F_{\lambda}=a \tau(\lambda) B_{\lambda}(T)+\beta F_{s o l a r}(\lambda)
$$

where $B_{\lambda}(T)$ is the Planck function, $F_{s o l a r}(\lambda)$ is the solar flux, and $\tau(\lambda)$ is the optical depth of a laboratory sample of dessicated $B$. coli (Vickramasinghe et al., 1986). Our result for $\mathrm{T}=320 \mathrm{~K}$ with $a: \beta$ chosen to give an optimal fit is shown in the solid curve of Fig. 2 . The dashed curve shows a more realistic model computed by Vallis et al. (1989) using data for irradiated $B$. coli and the cometary size distribution. A flux calculation using the resulting distribution of 


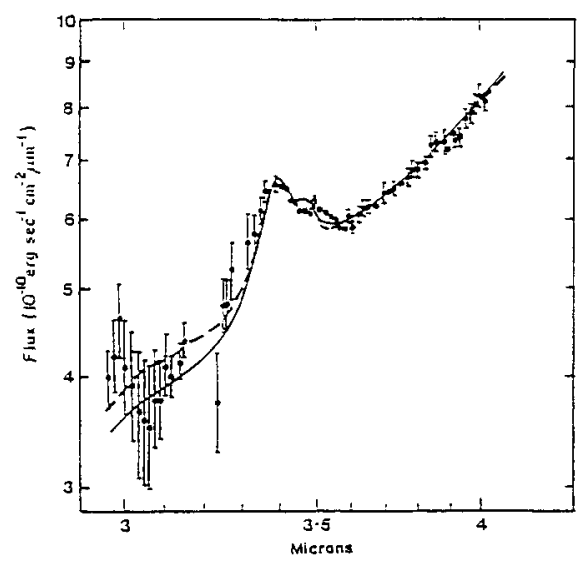

Fig. 2: Observational data for comet Halley on 1986 March 31 compared with calculations for cometary size distribution of organic grains (dashed curve). The solid curve is the prediction for a single grain temperature.

temperatures of the emitting grains is shown in the dashed curve of Fig. 2, for which agreement with the data is seen to be improved. Although the 2-4 $\mu \mathrm{m}$ infrared spectrum of comet Halley has shown a modest extent of variation from one observed date to another, the points in Fig. 2 may be taken as representative of dust emerging in a fresh eruption from the cometary surface. (The comet was seen to have brightened significantly on adjacent days around 1986 March 31.$)$ An interesting feature is that the $\tau(\lambda)$ data for an organic (biotic) sample, which was earlier found to give excellent agreement to the $2-4 \mu \mathrm{m}$ absorption spectrum of the galactic centre infrared source GC-IRS7, now matches the data for freshly evaporated cometary dust.

\section{Conclusion}

To conclude we note that close correspondences exist between the observed properties of cometary grains and interstellar dust over a wide range of wavelengths. The indications are for a generic

link between these grain types. If the direction of this link is from interstellar space to comets, it is indeed surprising to find a size distribution that has in the main been unmodified throughout the entire history of the solar nebula. Our preference is for organic (biotic) grains that are amplified within cometary objects in early solar nebula-type objects and subsequently injected into the interstellar medium. The cosmic amplification cycle of biotic grains is depicted in Fig. 3. Degradation products of such grains into aromatic and aliphatic hydrocarbons could account for the $3.28 \mu \mathrm{m}$ interstellar emission band and other spectral features in the mid-infrared wavelength region (Hoyle and Vickramasinghe, 1989; Wickramasinghe et al., 1990). It is interesting to note that 


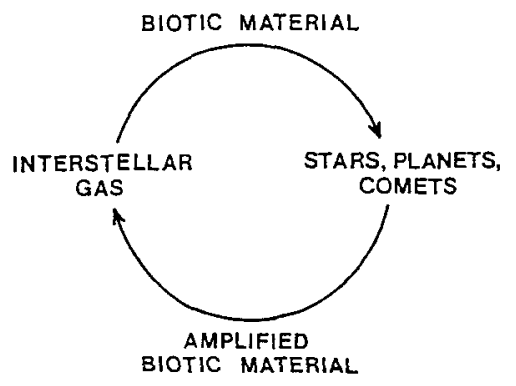

Fig. 3: Cosmic amplification cycle of biotic grains.

emission bands near $3.3 \mu \mathrm{m}$ and $11.25 \mu \mathrm{m}$ have also been observed in spectra of comet Halley (Hoyle and Vickramasinghe, 1989; Lynch et al., 1989).

\section{References}

Delsemme, A.H., (1976) in Lecture Notes in Physics, eds. H. Elsäser and H. Fechtig (Berline: Springer Verlag) Vol. 48, p.314.

Hoyle, F. and Vickramasinghe, N.C. (1986). Barth, Moon and Planets, 36, 289.

Hoyle, F. and Vickramasinghe, N.C. (1989). Proc. 22nd Bslab Symposium, ESA SP-290, 67.

Kissell, J. (1986). Nature, 321, 336.

Kissell, J. and Krueger, F.R. (1987). Nature, 326, 755.

Le Borgne, J.F., Leroy, J.L. and Arnaud, J. (1986) . 20th ESLAB Symp., ESA SP- 250, Vol. I, 571.

Lynch, D.K., Russe11, R.V., Campins, H., Vitteborn, F.C., Bregman, J.D., Rank, D.M. and Cohen, M. (1989). Icarus, 379.

Mazets, E.P. and 13 authors (1986). Nature, 321, 276.

McDonnel1, J.A.M. and 27 authors (1987). Istron. 1strophys., 187,719 .

Millman, P.I. (1976) in Lecture Notes in Physics, eds. H. Elsäser and H. Fechtig (Berlin: Springer Verlag) Vol. 48, p. 359-372.

Mukai, T., Mukai, S. and Kikuchi, S. (1987). Istron. 1strophys., 187, 650 .

Nandy, K. (1964). Publ. Roy. Obs. Bdin., 3, 142 and 4, 57.

Röser, S. (1976) in Lecture Notes in Physics, eds. H. Elsäer and H. Fechtig (Berlin: Springer Verlag) Vol. 48, p.319-322.

Vallis, K.K. and Vickramasinghe, N.C. (1990) in Evolution in 1strophysics (ESA SP-310).

Whipple, F. (1967) in The Zodiacal Light and the Interplanetary Yedium ed. J.L. Veinberg (NASA SP-150).

Vickramasinghe, N.C., Hoyle, P. and 11-Jabory, T. (1990). Istrophys. Sp. Sci., 166, 333.

Vickramasinghe, D.T., and Allen, D.A. (1986). Nature, 323, 44. 